\title{
Vibrational Studies of the Disulfide Group in Proteins. VI. General Correlations of SS and CS Stretch Frequencies with Disulfide Bridge Geometry
}

\author{
WEILI QIAN and SAMUEL KRIMM \\ Biophysics Research Division and Department of Physics, University of Michigan, Ann Arbor, Michigan 48109
}

\begin{abstract}
SYNOPSIS
Normal mode calculations have been done on a range of disulfide bridge conformations in order to determine how the SS stretch and CS stretch frequencies depend on structure. In addition to varying the $\mathrm{C}^{\alpha} \mathrm{C}^{\beta} \mathrm{SS}$ and $\mathrm{NC}^{\alpha} \mathrm{C}^{\beta} \mathrm{S}$ dihedral angles, we have varied the $\phi, \psi$ of the adjoining peptide groups since we have shown that these also influence the above frequencies. In order to obtain structural information from the observed frequencies, we have done a study of the conformational states found in 92 disulfide bridges in 25 known protein structures. This permits making a statistically based correlation between CS stretch frequencies and the possible contributing conformers. (c) 1992 John Wiley \& Sons, Inc.
\end{abstract}

\section{INTRODUCTION}

In previous papers of this series ${ }^{1-5}$ we developed the basis for a rigorous correlation of the frequencies of the Raman SS stretch, $\nu(\mathrm{SS})$, and CS stretch, $\nu(\mathrm{CS})$, bands with the geometry of the disulfide bridge. Such correlations can only be obtained from reliable normal mode analyses of the relevant structures, since empirical studies of simple model compounds ${ }^{6}$ can only provide rough general guidelines. We have sought to achieve such analyses by refining a scaled ab initio force field for the disulfide group, using experimental data available for alkyl disulfides. $^{1-3}$ This conformation-dependent force field was then combined with an empirical force field for the peptide chain ${ }^{7}$ to show that observed $\nu(\mathrm{SS})$ and $\nu$ (CS) Raman bands of $\mathrm{S}-\mathrm{S}$ bridges in known peptide and protein structures can be well reproduced. ${ }^{4}$ We also obtained a detailed correlation of $\nu$ (SS) with the CCSS dihedral angles from normal mode calculations on $92 \mathrm{~S}-\mathrm{S}$ bridges in 25 proteins of known structure. ${ }^{5}$

In this paper we present more general structurespectra correlations. We also explore the questions of what structural parameters of an $S-S$ bridge

Biopolymers, Vol. 32, 1025-1033 (1992)

(c) 1992 John Wiley \& Sons, Inc.

CCC 0006-3525/92/081025-09\$04.00 can be specified from knowledge of the $\nu(\mathrm{SS})$ and $\nu$ (CS) frequencies. This structure is determined in principle by five bridge dihedral angles, $\tau$, and (potentially) four peptide group angles, the two sets of $\phi, \psi$. [The former are $\tau\left(\mathrm{C}^{\beta} \mathrm{SSC}^{\beta}\right)\left(\chi^{3}\right)$, the two $\tau\left(\mathrm{C}^{\alpha} \mathrm{C}^{\beta} \mathrm{SS}\right)\left(\chi^{2}\right)$, and the two $\tau\left(\mathrm{NC}^{\alpha} \mathrm{C}^{\beta} \mathrm{S}\right)\left(\chi^{1}\right)$. The latter are $\tau\left(\mathrm{CNC}^{\alpha} \mathrm{C}\right)$ and $\tau\left(\mathrm{NC}^{\alpha} \mathrm{CN}\right)$, respectively.] Since the $\nu(\mathrm{SS})$ and $\nu(\mathrm{CS})$ represent at most three pieces of data, it is clear that the complete conformation of the bridge cannot be obtained uniquely from them alone. However, not all values of the dihedral angles are found to be equally probable, ${ }^{8}$ and we can use this information to significantly restrict the conformational space to be examined. In addition, data from actual protein structures suggest that certain combinations of allowed dihedral angles are more likely to be found than others. Thus, while the 9 dihedral angle parameters cannot be determined from three spectral frequencies, it turns out that, in light of the above kinds of restrictions, the observed $\nu$ (SS) and $\nu$ (CS) provide significant constraints on the allowable $S-S$ bridge conformations. We discuss below the detailed nature of these correlations.

\section{CALCULATIONS}

As in our previous studies, ${ }^{4,5}$ normal mode calculations were done on the unit 


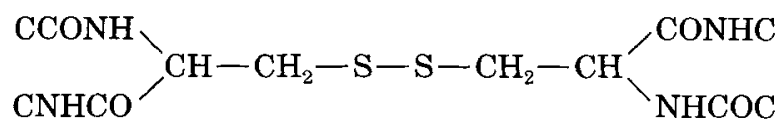

which was found to be the smallest model structure capable of giving a realistic representation of the vibrational frequencies of the $\mathrm{S}-\mathrm{S}$ bridge. As before, the force field consisted of two parts, the scaled $a b$ initio force field for the $S-S$ bridge refined from alkyl disulfides ${ }^{2,3}$ plus our empirical force field for the peptide group. ${ }^{7}$

Normal mode calculations for the entire range of the 9 dihedral angles would be prohibitive; fortunately, a limited set is in fact adequate. The $\chi^{3}$ angle is always found to be close to $+90^{\circ}$ (usually designated $G$ ) or $-90^{\circ}\left(\mathrm{G}^{\prime}\right)$ in proteins, ${ }^{8}$ and we have therefore restricted our calculations to this value. (Corrections to the SS stretch force constant for departures from this value are available. $\left.{ }^{3}\right)$ The $\chi^{2}$ angle is more variable, ${ }^{8}$ and in most cases we have examined the range of this angle at $30^{\circ}$ intervals: $0^{\circ}(\mathrm{C}), 30^{\circ}(\mathrm{A}), 60^{\circ}(\mathrm{G}), 90^{\circ}(\mathrm{B}), 120^{\circ}(\mathrm{S})$, $150^{\circ}(\mathrm{D}), 180^{\circ}(\mathrm{T})$, and their negative values $\left(\mathrm{D}^{\prime}\right.$, $\mathrm{S}^{\prime}, \mathrm{B}^{\prime}, \mathrm{G}^{\prime}$, and $\left.\mathrm{A}^{\prime}\right)$. The $\chi^{1}$ angles, as might be expected, are clustered about the standard rotamer values, ${ }^{8}$ and our calculations were done for these, designated by the atom $(\mathrm{N}, \mathrm{C}$, or $\mathrm{H})$ trans to $\mathrm{S}$ across the $\mathrm{C}^{\alpha} \mathrm{C}^{\beta}$ bond.

Our previous calculations on some known protein $\mathrm{S}-\mathrm{S}$ bridges ${ }^{4}$ already showed that $\nu(\mathrm{SS})$ and $\nu(\mathrm{CS})$ also depend on the $\phi, \psi$ of the adjacent peptide groups, and our present studies confirm this. Since the $\phi, \psi$ are restricted, however, we need not do calculations for all values. A previous examination of the $\phi, \psi$ associated with 72 known $\mathrm{S}-\mathrm{S}$ bridges ${ }^{8}$ determined the distribution with respect to adjoining peptide group conformations, and found that over half of the bridges linked $\beta$-strands $(\beta), \alpha$-helices $(\alpha)$, or involved both. We have done a similar analysis on the $92 \mathrm{~S}-\mathrm{S}$ bridges studied previously, ${ }^{5}$ and the results are shown in Figure 1. On this $60^{\circ}$ grid, we nominally associate square 1 with $\beta(\phi$ $\left.=-138.4^{\circ}, \psi=135.7^{\circ}\right)$ and 10 plus 11 with $\alpha(\phi$ $=-57.4^{\circ}, \psi=-47.5^{\circ}$ ). While square 2 has generally been considered also as $\beta$, we designate it as $\epsilon$ (based on an average of the observed angles, we characterize it as $\phi=-80^{\circ}, \psi=142^{\circ}$ ). We base this not only on the fact that the $\nu(\mathrm{CS})$ frequencies are significantly different from $\beta$, but also on the recognition that this "extended-helix" conformation ${ }^{9}$ is now accepted ${ }^{10}$ as an identifiable structure contributing to the CD spectra of proteins. Thus, most of the various calculations, discussed below in more detail,

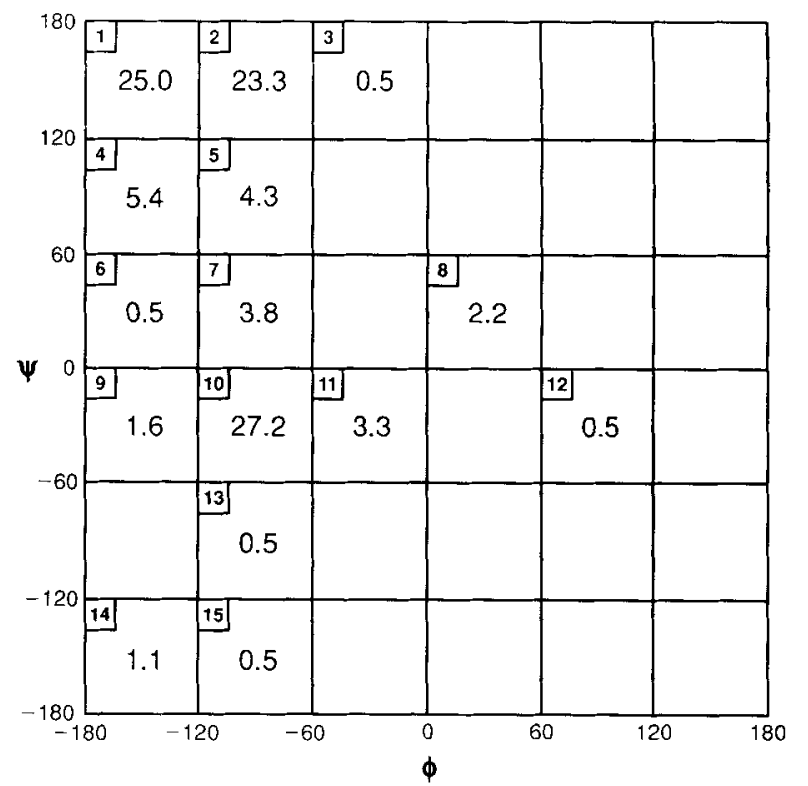

Figure 1. Distribution of $\phi, \psi$ for peptide groups of 92 disulfide bridges in 25 known protein structures. ${ }^{5}$ Large number is percentage of total conformations in grid box designated by small number.

have involved $\phi, \psi$ corresponding to the $\alpha, \beta$, and $\epsilon$ conformations.

The structures in our calculations all had $\chi^{3}$ $=+90^{\circ}$. For the comparable $\mathrm{S}-\mathrm{S}$ bridges with lefthanded chirality, i.e., $\mathrm{X}^{\prime} \mathrm{G}^{\prime} \mathrm{Y}^{\prime}$ for $\mathrm{XGY}$ (in the $\chi_{1}^{2} \chi^{3} \chi_{2}^{2}$ notation) but with the same $\phi, \psi$, we have verified that the $\nu(\mathrm{SS})$ differ by at most $2-3 \mathrm{~cm}^{-1}$.

\section{RESULTS AND DISCUSSION}

\section{Structure-Frequency Correlations}

SS Stretch Frequencies. Several considerations have entered into the determination of which structures to examine by normal mode calculations, in the context of keeping the number of calculations within manageable proportions. First, we found that, for $\nu$ (SS) frequencies, the distinction between $\beta$ and $\epsilon$ peptide conformations was minimal (averaging $\sim 2$ $\mathrm{cm}^{-1}$ ). Since these plus the $\alpha$ comprise about $80 \%$ of the observed $\phi, \psi$ of $S-S$ bridges in proteins ( see Figure 1), we have restricted our calculations to $\alpha$ and $\beta$ conformations. Second, we present the $\nu$ (SS) only for $\chi_{1}^{1}=\chi_{2}^{1}$, since calculations for mixed structures show that their frequencies are generally within $1-2 \mathrm{~cm}^{-1}$ of the average of the symmetrical structures. Third, while there is no regular variation of $\nu$ (CS) with $\chi^{2}$, this is the case for $\nu(\mathrm{SS})$. We 
therefore have calculated $\nu(\mathrm{CS})$ at $30^{\circ}$ intervals in $\chi^{2}$, but give $\nu(\mathrm{SS})$ only at $60^{\circ}$ intervals, together with figures for selected examples to demonstrate the detailed nature of the regular variation.

In Table I we present the $\nu$ (SS), with contributions $\geq 20$ to the potential energy distribution (PED), of $\mathbf{S}-\mathbf{S}$ bridges for which $\phi_{1}, \psi_{1}=\alpha$ and $\phi_{2}, \psi_{2}=\alpha$, i.e., the dihedral angles of the $\alpha$-helix. For each set of $\chi_{1}^{2} \chi_{2}^{2}$, values are given for $\chi_{1}^{1} \chi_{2}^{1}$ $=\mathrm{NN}, \mathrm{CC}$, and HH. Similar results are given in Table II for $\phi_{1}, \psi_{1}=\beta$ and $\phi_{2}, \psi_{2}=\beta$, and in Table III for $\phi_{1}, \psi_{1}=\alpha$ and $\phi_{2}, \psi_{2}=\beta$. In Figures 2 and 3 we show the more detailed variation of $\nu$ (SS) with $\chi_{1}^{2}=\chi_{2}^{2}$ for the $\alpha \alpha$ and $\beta \beta$ cases, respectively.

These results lead to several pertinent observations. First, as stated above, the $\nu$ (SS) clearly depend on the $\phi, \psi$ of the adjacent peptide groups, which was not predicted by model compound studies ${ }^{6}$ or approximate normal mode calculations. ${ }^{11}$ In fact, even for highly symmetrical $\chi_{1}^{1} \chi_{1}^{2} \chi_{2}^{2} \chi_{2}^{1}$ structures (we leave out the common $\chi^{3}$ angle in our designation of the bridge conformation), the results depend significantly on $\phi, \psi$; compare, for example, $517 \mathrm{~cm}^{-1}$ for $\alpha$ NGTN $\beta$ with $528 \mathrm{~cm}^{-1}$ for $\beta$ NGTN $\alpha$. Second, there is a significant dependence of $\nu(\mathrm{SS})$ on $\chi^{1}$, but with the order of frequencies for $\chi^{1}=\mathrm{N}, \mathrm{C}$, or
$H$ very much dependent on the $\phi, \psi$ as well as on $\chi^{2}$. Third, at least for $\chi_{1}^{2}=\chi_{2}^{2}$, it is clear (see Figures 2 and 3 ) that $\nu(\mathrm{SS})$ varies in a regular manner with $\chi^{2}$. Finally, it is important to note that in some cases a single $S-S$ bridge can give rise to two normal modes that contain contributions from SS stretch. We saw this for some specific peptide ${ }^{4}$ and protein $^{4,5}$ bridges, and we see now that it is associated with certain kinds of bridge conformations. In particular, splitting with significant $P E D$ contributions to both modes is found primarily for $\chi_{2}^{2}=\mathrm{T}, \chi_{1}^{1}=\chi_{2}^{1}=\mathrm{N}$, and especially for $\phi, \psi=\beta$. The above results thus provide a ready way of identifying the $\nu$ (SS) frequencies to be associated with a specified bridge geometry.

CS Stretch Frequencies. As we have already noted, ${ }^{2}$ the $\nu(\mathrm{CS})$ frequencies depend mainly on the geometry of the side of the $\mathrm{S}-\mathrm{S}$ bridge on which the $\mathrm{C}-\mathrm{S}$ bond is located. In Table IV we show this dependence on $\chi^{2}, \chi^{1}$, and $\phi, \psi$. Normal mode calculations were done at $30^{\circ}$ intervals in $\chi^{2}$, for the three standard rotamer values of $\chi^{1}$, and for $\phi, \psi$ $=\alpha, \beta$, and $\epsilon[$ for $\nu(\mathrm{CS}), \beta$ and $\epsilon$ conformations can give quite different frequencies]. Modes are given with $\mathrm{PED} \geq 15$, since in some cases more than one

Table I Disulfide Bridge SS Stretch Frequencies for $\phi_{1}, \psi_{1}=\phi_{2}, \psi_{2}=\alpha^{\mathrm{n}}$

\begin{tabular}{|c|c|c|c|c|c|c|c|}
\hline \multirow[b]{2}{*}{$\chi_{1}^{2 b}$} & \multirow[b]{2}{*}{$\chi_{1}^{1} \chi_{2}^{1}{ }^{\mathrm{c}}$} & \multicolumn{6}{|c|}{$\chi_{2}^{2 b}$} \\
\hline & & $\mathrm{T}$ & $\mathrm{S}^{\prime}$ & $\mathrm{G}^{\prime}$ & $\mathrm{C}$ & G & S \\
\hline \multirow[t]{3}{*}{$\mathbf{T}$} & NN & $537(54) 555(32)^{d}$ & & & & & \\
\hline & $\mathrm{CC}$ & $534(85)$ & & & & & \\
\hline & $\mathrm{HH}$ & $529(79)$ & & & & & \\
\hline \multirow[t]{3}{*}{$\mathbf{S}^{\prime}$} & $\mathrm{NN}$ & $531(70)$ & $520(83)$ & & & & \\
\hline & $\mathrm{CC}$ & $523(80)$ & $512(86)$ & & & & \\
\hline & $\mathrm{HH}$ & $521(83)$ & $511(89)$ & & & & \\
\hline \multirow[t]{3}{*}{$\mathrm{G}^{\prime}$} & $\mathrm{NN}$ & $525(76)$ & $510(90)$ & $503(90)$ & & & \\
\hline & $\mathrm{CC}$ & $518(82)$ & $506(88)$ & $501(87)$ & & & \\
\hline & $\mathbf{H H}$ & $517(83)$ & $506(92)$ & $502(92)$ & & & \\
\hline \multirow[t]{3}{*}{$\mathrm{C}$} & $\mathrm{NN}$ & $533(66)$ & $523(78)$ & $516(79)$ & $526(75)$ & & \\
\hline & $\mathrm{CC}$ & $527(79)$ & $516(81)$ & $511(83)$ & $519(79)$ & & \\
\hline & $\mathrm{HH}$ & $525(80)$ & $515(86)$ & $511(87)$ & $520(83)$ & & \\
\hline \multirow[t]{3}{*}{ G } & NN & $527(72)$ & $514(84)$ & $503(92)$ & $517(79)$ & $508(90)$ & \\
\hline & $\mathrm{CC}$ & $523(90)$ & $510(92)$ & $503(93)$ & $514(89)$ & $508(99)$ & \\
\hline & $\mathrm{HH}$ & $520(84)$ & $508(92)$ & $503(95)$ & $513(89)$ & $506(97)$ & \\
\hline \multirow[t]{3}{*}{ S } & $\mathrm{NN}$ & $527(62) 554(23)$ & $516(78)$ & $509(83)$ & $520(72)$ & $512(83)$ & $515(74)$ \\
\hline & $\mathrm{CC}$ & $526(84)$ & $515(86)$ & $509(86)$ & $518(83)$ & $514(93)$ & $519(86)$ \\
\hline & $\mathrm{HH}$ & $523(83)$ & $513(88)$ & $509(88)$ & $517(86)$ & $512(91)$ & $516(87)$ \\
\hline
\end{tabular}

${ }^{a}$ Dihedral angles of $\alpha$-helix $\left(\phi=-57.4^{\circ}, \psi=-47.5^{\circ}\right)$.

${ }^{b} \chi^{2}=\tau\left(\mathrm{C}^{\alpha} \mathrm{C}^{\beta} \mathrm{SS}\right) . \mathrm{T}=180^{\circ}, \mathrm{S}=120^{\circ}, \mathrm{G}=60^{\circ}, \mathrm{C}=0^{\circ}$; prime indicates negative angle.

${ }^{c} \chi^{1}=\tau\left(\mathrm{NC}^{\alpha} \mathrm{C}^{\beta} \mathrm{S}\right) . \mathrm{N}, \mathrm{C}, \mathrm{H}$ are atoms trans to $\mathrm{S}$.

d Potential energy distribution in SS stretch $\geq 20$. 
Table II Disulfide Bridge SS Stretch Frequencies for $\phi_{1}, \psi_{1}=\phi_{2}, \psi_{2}=\beta^{\mathrm{a}}$

\begin{tabular}{|c|c|c|c|c|c|c|c|}
\hline \multirow[b]{2}{*}{$\chi_{1}^{2 b}$} & \multirow[b]{2}{*}{$\chi_{1}^{1} \chi_{2}^{1 c}$} & \multicolumn{6}{|c|}{$\chi_{2}^{2 b}$} \\
\hline & & $\mathrm{T}$ & $\mathrm{S}^{\prime}$ & $\mathrm{G}^{\prime}$ & $\mathrm{C}$ & $\mathrm{G}$ & $\mathrm{S}$ \\
\hline \multirow[t]{3}{*}{$\mathrm{T}$} & NN & $521(40) 554(50)^{\mathrm{d}}$ & & & & & \\
\hline & $\mathrm{CC}$ & $527(51) 550(39)$ & & & & & \\
\hline & $\mathrm{HH}$ & $527(55) 563(27)$ & & & & & \\
\hline \multirow[t]{3}{*}{$\mathrm{S}^{\prime}$} & $\mathrm{NN}$ & $516(53) 551(38)$ & $509(69) 547(25)$ & & & & \\
\hline & $\mathrm{CC}$ & 522 & $515(92)$ & & & & \\
\hline & $\mathrm{HH}$ & $518(62) 562(21)$ & $507(74)$ & & & & \\
\hline \multirow[t]{3}{*}{$\mathrm{G}^{\prime}$} & $\mathrm{NN}$ & $515(63) 546(26)$ & $506(83)$ & $503(91)$ & & & \\
\hline & $\mathrm{CC}$ & $518(74)$ & $508(91)$ & $503(86)$ & & & \\
\hline & $\mathrm{HH}$ & $518(69)$ & $504(84)$ & $503(87)$ & & & \\
\hline \multirow[t]{3}{*}{$\mathrm{C}$} & NN & $523(46) 547(39)$ & $516(69) 543(21)$ & $512(85)$ & $526(85)$ & & \\
\hline & $\mathrm{CC}$ & $526(62) 546(24)$ & $519(83)$ & $513(83)$ & $523(80)$ & & \\
\hline & $\mathrm{HH}$ & $524(64)$ & $513(74)$ & $512(81)$ & $520(79)$ & & \\
\hline \multirow[t]{3}{*}{ G } & NN & $518(59) 547(29)$ & $509(79)$ & $504(93)$ & $517(86)$ & $509(93)$ & \\
\hline & $\mathrm{CC}$ & $520(7) 545(20)$ & $511(91)$ & $503(90)$ & $515(84)$ & $507(92)$ & \\
\hline & $\mathrm{HH}$ & $520(69)$ & $506(83)$ & $503(94)$ & $514(85)$ & $506(98)$ & \\
\hline \multirow[t]{3}{*}{$S$} & NN & $517(53) 551(38)$ & $509(70) 547(25)$ & $506(81)$ & $516(70) 544(21)$ & $510(79)$ & $511(69) 550(27)$ \\
\hline & $\mathrm{CC}$ & $518(53) 548(34)$ & $511(72) 541(22)$ & $506(73)$ & $515(64)$ & $509(75) 542(21)$ & $510(61) 546$ \\
\hline & $\mathrm{HH}$ & $524(65)$ & $512(75)$ & $512(80)$ & $519(79)$ & 514 & $519(76)$ \\
\hline
\end{tabular}

${ }^{\mathrm{a}}$ Dihedral angles of $\beta$-sheet $\left(\phi=-138.4^{\circ}, \psi=135.7^{\circ}\right)$.

${ }^{\mathrm{b}} \chi^{2}=\tau\left(\mathrm{C}^{\alpha} \mathrm{C}^{\beta} \mathrm{SS}\right) . \mathrm{T}=180^{\circ}, \mathrm{S}=120^{\circ}, \mathrm{G}=60^{\circ}, \mathrm{C}=0^{\circ}$; prime indicates negative angle.

${ }^{c} \chi^{1}=\tau\left(\mathrm{NC}^{\alpha} \mathrm{C}^{\theta} \mathrm{S}\right) . \mathrm{N}, \mathrm{C}, \mathrm{H}$ are atoms trans to $\mathrm{S}$.

d Potential energy distribution in SS stretch $\geq 20$.

Table III Disulfide Bridge SS Stretch Frequencies for $\phi_{1}, \psi_{1}=\alpha, \phi_{2}, \psi_{2}=\beta^{\mathrm{a}}$

\begin{tabular}{|c|c|c|c|c|c|c|c|}
\hline \multirow[b]{2}{*}{$\chi_{1}^{2 b}$} & \multirow[b]{2}{*}{$\chi_{1}^{1} \chi_{2}^{1} \mathbf{c}$} & \multicolumn{6}{|c|}{$\chi_{2}^{2 b}$} \\
\hline & & $\mathrm{T}$ & $\mathbf{S}^{\prime}$ & $G^{\prime}$ & $\mathrm{C}$ & G & $\mathrm{S}$ \\
\hline \multirow[t]{3}{*}{$\mathrm{T}$} & NN & $526(35) 553(37)^{\mathrm{d}}$ & $524(50)$ & $524(70)$ & $534(67)$ & $528(72)$ & $524(51) 552(20)$ \\
\hline & $\mathrm{CC}$ & $529(56) 545(27)$ & $525(78)$ & $519(84)$ & $529(80)$ & $522(79)$ & $519(55) 543(34)$ \\
\hline & $\mathrm{HH}$ & $528(64)$ & $518(73)$ & $517(83)$ & $525(78)$ & $520(84)$ & $524(79)$ \\
\hline \multirow[t]{3}{*}{$\mathbf{S}^{\prime}$} & NN & $520(53) 550(20)$ & $514(71)$ & $510(88)$ & $522(83)$ & $515(86)$ & $514(72)$ \\
\hline & $\mathrm{CC}$ & $521(69) 546(22)$ & $513(89)$ & $507(88)$ & $517(81)$ & $509(89)$ & $510(72)$ \\
\hline & $\mathrm{HH}$ & $521(69)$ & $509(80)$ & $506(91)$ & $516(83)$ & $508(93)$ & $515(83)$ \\
\hline \multirow[t]{3}{*}{$\mathrm{G}^{\prime}$} & NN & $515(64) 543(20)$ & $505(83)$ & $503(90)$ & $515(87)$ & $504(94)$ & $506(82)$ \\
\hline & $\mathrm{CC}$ & $516(73)$ & $507(90)$ & $502(87)$ & $512(82)$ & $502(90)$ & $505(74)$ \\
\hline & $\mathrm{HH}$ & $518(69)$ & $504(85)$ & $502(89)$ & $512(82)$ & $503(95)$ & $512(80)$ \\
\hline \multirow[t]{3}{*}{$\mathrm{C}$} & NN & $523(45) 550(26)$ & $517(64)$ & $516(78)$ & $526(80)$ & $518(80)$ & $517(65)$ \\
\hline & $\mathrm{CC}$ & $524(64)$ & $517(83)$ & $512(83)$ & $521(80)$ & $513(84)$ & $514(66) 541(20)$ \\
\hline & $\mathrm{HH}$ & $524(66)$ & $512(77)$ & $511(86)$ & $520(81)$ & $513(89)$ & $519(80)$ \\
\hline \multirow[t]{3}{*}{ G } & NN & $517(58)$ & $509(78)$ & $504(92)$ & $517(85)$ & $508(91)$ & $509(79)$ \\
\hline & $\mathrm{CC}$ & $521(76)$ & $512(95)$ & $504(94)$ & $516(89)$ & $508(95)$ & $510(77)$ \\
\hline & $\mathrm{HH}$ & $520(69)$ & $506(83)$ & $503(94)$ & $513(85)$ & $506(98)$ & $514(85)$ \\
\hline \multirow[t]{3}{*}{$\mathrm{S}$} & NN & $518(55) 554(28)$ & $511(72)$ & $509(82)$ & $519(77)$ & $512(83)$ & $513(70)$ \\
\hline & $\mathrm{CC}$ & $527(85)$ & $511(67) 531(24)$ & $510(87)$ & $520(84)$ & $513(88)$ & $514(67) 541(20)$ \\
\hline & $\mathrm{HH}$ & $522(69)$ & $510(80)$ & $509(87)$ & $517(84)$ & $512(92)$ & $518(81)$ \\
\hline
\end{tabular}

${ }^{\mathrm{a}}$ Dihedral angles of $\alpha$-helix $\left(\phi=-57.4^{\circ}, \psi=-47.5^{\circ}\right)$ and $\beta$-sheet $\left(\phi=-138.4^{\circ}, \psi=135.7^{\circ}\right)$.

${ }^{\mathrm{b}} \chi^{2}=\tau\left(\mathrm{C}^{\alpha} \mathrm{C}^{\beta} \mathrm{SS}\right) . \mathrm{T}=180^{\circ}, \mathrm{S}=120^{\circ}, \mathrm{G}=60^{\circ}, \mathrm{C}=0^{\circ}$; prime indicates negative angle.

c $\chi^{1}=\tau\left(\mathrm{NC}^{\alpha} \mathrm{C}^{\beta} \mathrm{S}\right) . \mathrm{N}, \mathrm{C}, \mathrm{H}$ are atoms trans to $\mathrm{S}$.

${ }^{d}$ Potential energy distribution in SS stretch $\geq 20$. 


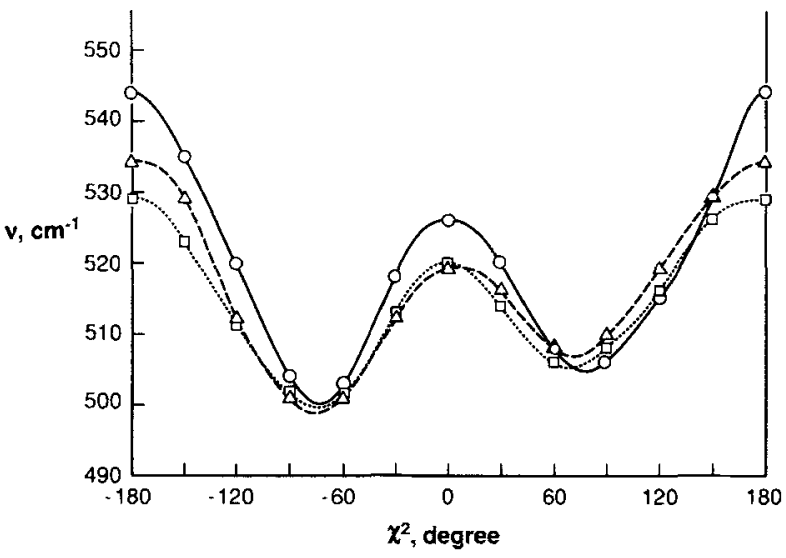

Figure 2. Variation of SS stretch frequency with $\chi^{2}\left(\chi_{1}^{2}=\chi_{2}^{2}\right)$ and $\chi_{1}^{1}=\chi_{2}^{1}=N(O), C(\Delta)$, and $H(\square)$ for $\phi_{1}, \psi_{1}=\phi_{2}, \psi_{2}=\alpha$. Where contributions occur to two normal modes, the PED-weighted frequency is given.

contribution is found near 20 , which is comparable to that over 20.

As can be seen from Table IV, the $\nu$ (CS) of a given bond generally makes contributions to more than one normal mode in the $600-850-\mathrm{cm}^{-1}$ region. In many cases one $\nu$ (CS) mode dominates, but in others the contributions to two or three modes can be comparable. The former situation predominates for $\chi^{1}=\mathrm{N}$ while the latter predominates for $\chi^{1}=\mathrm{C}$.

\section{Spectra-Structure Correlations}

If an $\mathrm{S}-\mathrm{S}$ bridge structure is explicitly known, we have shown that our disulfide plus peptide force fields permit a reliable normal mode calculation of its specific $\nu(\mathrm{SS})$ and $\nu(\mathrm{CS})$ frequencies. ${ }^{4}$ Or if the general bridge parameters are known, Tables I-IV can, in certain common cases, be used to determine the values of these frequencies.

It is often desirable, however, to reverse the process, viz., to obtain information on the structure from the spectrum. As we pointed out above, this is not easily achievable since the maximum of three observable stretching frequencies of a bridge are determined by 9 dihedral angles. (Of course, we could include other bands determined by the bridge geometry, such as angle bend and torsion modes. The problem is that they occur at lower frequencies and are harder to identify, since they mix more extensively with other coordinates as well as being overlapped in the spectrum by other deformation modes of the molecule.) A glance at Table IV, for example, shows how difficult it would be to determine $\chi^{2}$ and even the limited $\phi, \psi$ in the relatively well-defined
$\chi^{1}=\mathrm{N}$ domain because of the extensive frequency overlap.

While we should not expect this problem to be solvable, it is worth inquiring whether other considerations might restrict the total range of possibilities. In other words, just as the $\chi_{1}^{2} \chi_{2}^{2}$ combinations found in $92 \mathrm{~S}-\mathrm{S}$ bridges of proteins of known structure fall into a limited number of types, ${ }^{5}$ it can be expected that not all $\chi^{2} \chi^{1} \phi, \psi$ combinations are found with equal probability. And just as the $\chi_{1}^{2} \chi_{2}^{2}$ limitation led to constraints on the conformations that could be consistent with observed $\nu$ (SS) bands, ${ }^{5}$ analogous observed restrictions on $\chi^{2} \chi^{1} \phi, \psi$ would reduce the range of possible conformations deducible from the observed $\nu(\mathrm{CS})$ over that represented in Table IV. Such a statistical approach, while not uniquely defining a structure from the spectrum, may nevertheless be useful as a guide to likely possibilities.

We approach this problem as follows. First, we eliminate from consideration those $\phi, \psi$ regions (viz., squares in Figure 1) that contain less than $2 \%$ of the total $\chi^{2} \chi^{1} \phi, \psi$ conformations. This leaves 174 , or $95 \%$, of the $\mathrm{C}-\mathrm{S}$ conformers of the $92 \mathrm{~S}-\mathrm{S}$ bridges that we have studied. ${ }^{5}$ Second, we have determined the distribution of their $\chi^{2} \chi^{1} \phi, \psi$, and this is given in Table V. As can be seen, this distribution is distinctly nonuniform. Third, from normal mode calculations as a function of $\phi$ and $\psi$, we have ascertained that $\nu(\mathrm{CS})$ frequencies for comparable $\chi^{2} \chi^{1}$ conformers with $\phi, \psi$ of square 4 (Figure 1 ) are within a few $\mathrm{cm}^{-1}$ of those of square 1 , and similarly for squares 5 and 2 . We therefore use the calculated $\beta$ and $\epsilon$ frequencies of Table IV as representative of these respective pairs of $\phi, \psi$. In addition to the al-

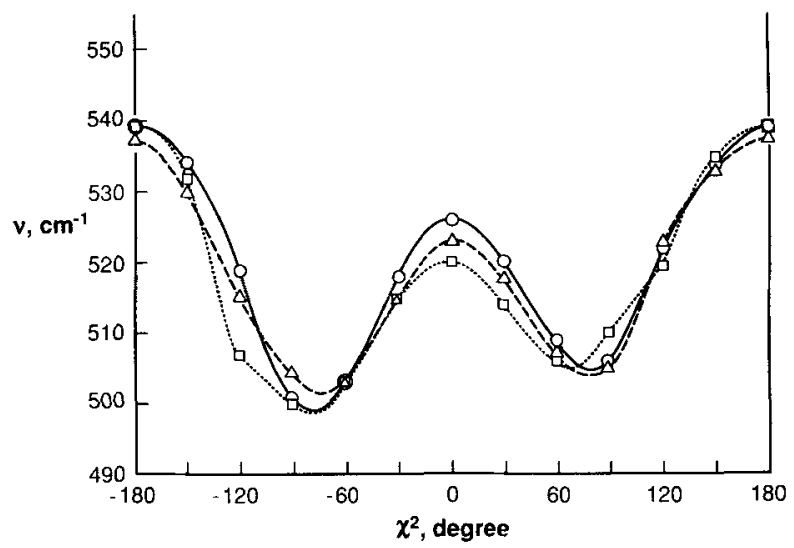

Figure 3. Variation of SS stretch frequency with $\chi^{2}\left(\chi_{1}^{2}=\chi_{2}^{2}\right)$ and $\chi_{1}^{1}=\chi_{2}^{1}=N(O), C(\triangle)$, and $H(\square)$ for $\phi_{1}, \psi_{1}=\phi_{2}, \psi_{2}=\beta$. Where contributions occur to two normal modes, the PED-weighted frequency is given. 
Table IV Disulfide Bridge CS Stretch Frequencies

\begin{tabular}{|c|c|c|c|c|c|c|c|c|c|}
\hline \multirow[b]{2}{*}{$\chi^{2 \mathbf{b}}$} & \multicolumn{9}{|c|}{$\chi^{1} \phi, \psi^{a}$} \\
\hline & $\mathrm{N} \alpha$ & $\mathrm{N} \beta$ & $\mathrm{N} \epsilon$ & $\mathrm{C} \alpha$ & $\mathrm{C} \beta$ & $\mathrm{C} \epsilon$ & $\mathrm{H} \alpha$ & $\mathrm{H} \beta$ & $\mathrm{H} \epsilon$ \\
\hline \multirow[t]{3}{*}{$\mathrm{T}$} & $751(65)^{\mathrm{c}}$ & $777(60)$ & $757(66)$ & $837(28)$ & $814(28)$ & $803(15)$ & $762(20)$ & $673(52)$ & $699(16)$ \\
\hline & & & & $686(17)$ & $707(33)$ & $725(29)$ & $687(30)$ & & $668(45)$ \\
\hline & & & & $651(23)$ & & $666(20)$ & & & \\
\hline \multirow[t]{3}{*}{$\mathrm{D}^{\prime}$} & $741(67)$ & $769(60)$ & $747(68)$ & $830(24)$ & $807(26)$ & $721(23)$ & $683(34)$ & $669(57)$ & $666(46)$ \\
\hline & & & & $682(20)$ & $705(34)$ & $665(23)$ & $617(24)$ & & \\
\hline & & & & $651(25)$ & $673(15)$ & & & & \\
\hline \multirow[t]{3}{*}{$\mathbf{S}^{\prime}$} & $731(68)$ & $759(57)$ & $736(69)$ & $826(21)$ & $800(23)$ & 799 (19) & $678(37)$ & $664(62)$ & $661(58)$ \\
\hline & & & & $678(31)$ & $702(33)$ & $718(32)$ & $616(19)$ & & \\
\hline & & & & $649(28)$ & $671(19)$ & 664 (18) & $604(15)$ & & \\
\hline \multirow[t]{3}{*}{$\mathbf{B}^{\prime}$} & $728(69)$ & $756(56)$ & $732(70)$ & $823(19)$ & $796(22)$ & $796(20)$ & $676(39)$ & $663(38)$ & $662(64)$ \\
\hline & & & & $677(25)$ & $703(34)$ & $718(33)$ & $618(23)$ & & \\
\hline & & & & $652(27)$ & $672(20)$ & $664(25)$ & & & \\
\hline \multirow[t]{3}{*}{$\mathrm{G}^{\prime}$} & $725(69)$ & $753(54)$ & $730(69)$ & $821(19)$ & $795(21)$ & 794 (19) & $676(38)$ & $663(69)$ & $662(66)$ \\
\hline & & & & $676(30)$ & $704(33)$ & $717(34)$ & & & \\
\hline & & & & $655(29)$ & $672(21)$ & $664(23)$ & & & \\
\hline \multirow[t]{3}{*}{$\mathrm{A}^{\prime}$} & $720(67)$ & $747(54)$ & $724(53)$ & $819(17)$ & $792(19)$ & $791(17)$ & $673(32)$ & $656(64)$ & $655(57)$ \\
\hline & & & $721(17)$ & $676(18)$ & $701(30)$ & $715(33)$ & $616(28)$ & & \\
\hline & & & & $652(39)$ & $671(24)$ & $662(28)$ & $603(21)$ & & \\
\hline \multirow[t]{3}{*}{$\mathrm{C}$} & $715(60)$ & $741(49)$ & $715(55)$ & $648(46)$ & $790(17)$ & $713(31)$ & $670(18)$ & $649(52)$ & $650(41)$ \\
\hline & & & & & $697(26)$ & $660(29)$ & $614(22)$ & $615(21)$ & $636(18)$ \\
\hline & & & & & $669(26)$ & & $600(36)$ & & $625(15)$ \\
\hline \multirow[t]{3}{*}{ A } & $722(65)$ & $745(55)$ & $726(45)$ & $676(18)$ & $792(19)$ & $790(15)$ & $673(34)$ & $655(64)$ & $654(56)$ \\
\hline & & & $717(25)$ & $651(39)$ & $700(33)$ & $717(33)$ & $617(28)$ & $618(17)$ & \\
\hline & & & & & $672(22)$ & $663(27)$ & 603 (19) & & \\
\hline \multirow[t]{3}{*}{ G } & $729(66)$ & $747(60)$ & $731(56)$ & $677(30)$ & $793(20)$ & $720(37)$ & $676(38)$ & $659(68)$ & $657(60)$ \\
\hline & & & $713(16)$ & $653(31)$ & $701(36)$ & $666(20)$ & $619(22)$ & & \\
\hline & & & & & $674(18)$ & & & & \\
\hline \multirow[t]{3}{*}{$\mathrm{B}$} & $730(61)$ & $749(60)$ & $732(57)$ & $676(25)$ & 794 (19) & $720(36)$ & $676(38)$ & $658(69)$ & $657(62)$ \\
\hline & & & $713(15)$ & $652(30)$ & $701(35)$ & $665(24)$ & $618(27)$ & & \\
\hline & & & & & $673(18)$ & & & & \\
\hline \multirow[t]{3}{*}{$\mathrm{S}$} & $732(67)$ & $751(59)$ & $734(59)$ & $828(22)$ & $794(16)$ & $720(33)$ & $677(33)$ & $657(67)$ & $656(59)$ \\
\hline & & & & $678(16)$ & $700(33)$ & $664(24)$ & $617(24)$ & & \\
\hline & & & & $650(31)$ & $673(19)$ & & $603(15)$ & & \\
\hline \multirow[t]{3}{*}{$\mathrm{D}$} & $741(67)$ & $763(61)$ & $745(64)$ & $833(25)$ & $802(18)$ & $819(15)$ & $757(17)$ & $666(62)$ & $662(49)$ \\
\hline & & & & $683(21)$ & $704(34)$ & $722(31)$ & $682(33)$ & & \\
\hline & & & & $651(26)$ & $674(15)$ & $665(22)$ & $617(23)$ & & \\
\hline
\end{tabular}

${ }^{\mathrm{a}} \chi^{1}=\tau\left(\mathrm{NC} \mathrm{C}^{\alpha} \mathrm{S}\right) . \mathrm{N}, \mathrm{C}, \mathrm{H}$ are atoms trans to S. $\alpha=\alpha$-helix $\left(\phi=-57.4^{\circ}, \psi=-47.5^{\circ}\right), \beta=\beta$-sheet $\left(\phi=-138.4^{\circ}, \psi=135.7^{\circ}\right), \epsilon$ $=$ "extended helix" $\left(\phi=-80^{\circ}, \psi=142^{\circ}\right)$.

${ }^{\mathrm{b}} \chi^{2}=\tau\left(\mathrm{C}^{\alpha} \mathrm{C}^{\beta} \mathrm{SS}\right) . \mathrm{T}=180^{\circ}, \mathrm{D}=150^{\circ}, \mathrm{S}=120^{\circ}, \mathrm{B}=90^{\circ}, \mathrm{G}=60^{\circ}, \mathrm{A}=30^{\circ}, \mathrm{C}=0^{\circ}$; prime indicates negative angle

${ }^{c}$ Potential energy distribution in $\mathrm{CS}$ stretch $\geq 15$.

ready calculated $\alpha$ frequencies for squares $10+11$, separate calculations were done for the conformers represented by square 7 (designated by $\alpha \beta$ ) and by square 8 (designated by $\alpha_{\mathrm{L}}$ ). Fourth, we use these calculations to provide the $\nu(\mathrm{CS})$ frequencies associated with the observed conformers of Table $\mathrm{V}$, with the provisos that marginal frequencies (with PED $\sim 20$ ) are excluded as well as those associated with only one conformer (except if they are part of
$1+4,2+5$, or $10+11$ pairs). This reduces the number of conformers in the pool to 154 , or $84 \%$ of the total, but includes essentially all conformers for which 2 or more examples are found in the $92 \mathrm{~S}-\mathrm{S}$ bridges of proteins. This selection process indicates that only $18 \%$ of the total possible number of $\chi^{2} \chi^{1}$ $\phi, \psi\left(=\alpha, \beta, \epsilon, \alpha \beta, \alpha_{\mathrm{L}}\right)$ combinations are actually found with a significant frequency in proteins. Finally, by collecting the conformers contributing to 
Table V Distribution of $\chi^{2} \chi^{1} \phi, \psi$ Among $174 \mathrm{C}-\mathrm{S}$ Conformers of Disulfide Bridges in Proteins

\begin{tabular}{|c|c|c|c|c|c|c|c|c|c|c|c|c|c|}
\hline \multirow[b]{2}{*}{$\chi^{1 \mathrm{~b}}$} & \multirow[b]{2}{*}{$\phi, \psi^{c}$} & \multicolumn{12}{|c|}{$x^{2 \mathrm{a}}$} \\
\hline & & $\mathrm{T}$ & $\mathrm{D}^{\prime}$ & $\mathrm{S}^{\prime}$ & $\mathrm{B}^{\prime}$ & $\mathrm{G}^{\prime}$ & $\mathrm{A}^{\prime}$ & C & A & $\mathrm{G}$ & B & $\mathrm{S}$ & $\mathrm{D}$ \\
\hline \multirow[t]{8}{*}{$\mathrm{N}$} & 1 & 1 & & & & & & & & 5 & 1 & 1 & \\
\hline & 2 & & & & & & & & & 2 & & & \\
\hline & 4 & 2 & & & & & & & & 1 & 1 & 2 & 1 \\
\hline & 5 & 2 & & & & & & & & & & & \\
\hline & 7 & & & & 1 & & & & & & & & \\
\hline & 8 & & & & & & & & & & & & \\
\hline & 10 & 2 & 2 & & 1 & & & & & 7 & 6 & & \\
\hline & 11 & & & & & & & & & & 1 & & \\
\hline \multirow[t]{8}{*}{$\mathrm{C}$} & 1 & 1 & & 4 & 12 & 1 & & & 1 & 5 & 8 & & \\
\hline & 2 & 7 & & 2 & 2 & 6 & & & & 11 & 7 & 2 & 1 \\
\hline & 4 & & 1 & 1 & & & & & & & 1 & & \\
\hline & 5 & & & & & 2 & & & & 1 & 1 & & \\
\hline & 7 & & & & & & & & & 3 & & & 2 \\
\hline & 8 & 3 & & & & & & & & & & & \\
\hline & 10 & 1 & & & 1 & 3 & & & 2 & 13 & 1 & & 9 \\
\hline & 11 & & & 1 & 1 & & & & & 1 & & & \\
\hline \multirow[t]{8}{*}{$\mathrm{H}$} & 1 & 1 & 1 & 1 & & & & & & & 2 & 1 & \\
\hline & 2 & & & & & & & & & & & & 3 \\
\hline & 4 & & & & & & & & & & & & \\
\hline & 5 & 1 & & & & & & & & & 1 & & \\
\hline & 7 & & & & & & & & & & 1 & & \\
\hline & 8 & 1 & & & & & & & & & & & \\
\hline & 10 & & & & & & & & & & 2 & & \\
\hline & 11 & & & & & & & & & & 1 & & 1 \\
\hline
\end{tabular}

${ }^{\circ} \chi^{2}=\tau\left(\mathrm{C}^{\alpha} \mathrm{C}^{\beta} \mathrm{SS}\right) . \mathrm{T}=180^{\circ}, \mathrm{D}=150^{\circ}, \mathrm{S}=120^{\circ}, \mathrm{B}=90^{\circ}, \mathrm{G}=60^{\circ}, \mathrm{A}=30^{\circ}, \mathrm{C}=0^{\circ} ;$ prime indicates negative angle.

${ }^{\text {h }} \chi^{1}=\tau\left(\mathrm{NC}^{\alpha} \mathrm{C}^{\beta} \mathrm{S}\right) . \mathrm{N}, \mathrm{C}, \mathrm{H}$ indicate atoms trans to $\mathrm{S}$.

' Numbers refer to squares in Figure 1.

each frequency region, we arrive at the frequencystructure correlation of Table VI.

We hasten to point out that, of course, this correlation is not unique, since it is based on a statistical weighting of conformations in observed $S-S$ bridges. Other structures, not found or excluded because of their low incidence in our sample, could contribute to $\nu(\mathrm{CS})$ frequencies in a given region. Because of small variations in dihedral angles from the "canonical" structures computed here, frequencies could vary by a few and perhaps up to $5 \mathrm{~cm}^{-1}$, leading to overlapping conformational assignments. Nevertheless, the above relationships may represent a useful narrowing of the likely possibilities that are consistent with the observed spectra.

Within the confines of the above approach, Table VI reveals some interesting correlations. Some bands (or band pairs) are relatively uniquely associated with single conformations. This is particularly true for most $\chi^{2}=\mathrm{T}$ structures, where band overlap is minimal. In several cases $(664-5+717-20,701-3$,
$729-31$ ), the possible $\chi^{2}$ are within $30^{\circ}$ ranges of each other, so that if such a fine distinction is not made the structural determination is even stronger. In the first two of these cases, as well as two others (652-5 + 676-7 and 718-25), the $\chi^{1} \phi, \psi$ of all conformers are the same, which means that this part of the structure can be strongly inferred. And in the other case (729-31), two of the three $\chi^{1} \phi, \psi$ are the same, as is the case for 3 of the 4 conformers contributing to the $747-751-\mathrm{cm}^{-1}$ region. Thus, determination of the $\nu(\mathrm{CS})$ frequencies can provide significant detailed information on the $\chi^{2} \chi^{1} \phi, \psi \mathrm{pa}$ rameters of an $\mathrm{S}-\mathrm{S}$ bridge.

\section{CONCLUSIONS}

In a previous paper, ${ }^{4}$ we showed that frequencies of observed $\nu$ (SS) and $\nu(\mathrm{CS})$ Raman bands of $\mathrm{S}-\mathrm{S}$ bridges in proteins of known structure could be satisfactorily reproduced by normal mode calculations 
Table VI CS Stretch Frequency-Structure Correlations for Disulfide Bridges ${ }^{a}$

\begin{tabular}{llr}
\hline Spectral Region & \multicolumn{1}{c}{ Contributing Conformers } & Total \\
\hline $618+676$ & $\mathrm{BH} \alpha(3)$ & 3 \\
651 & $\mathrm{AC} \alpha(2)$ & 2 \\
$651+833$ & $\mathrm{DC} \alpha(9)$ & 9 \\
$652-655+676-677$ & $\mathrm{~B} C \mathrm{C} \alpha(2) \mathrm{G} \mathrm{C} \alpha(3) \mathrm{GC} \alpha(14)$ & 19 \\
$657-8$ & $\mathrm{GC} \alpha \beta(3) \mathrm{BH} \beta(2)$ & 5 \\
$662-3$ & $\mathrm{DH} \epsilon(3) \mathrm{DC} \alpha \beta(2)$ & 5 \\
$664-665+717-720$ & $\mathrm{~B}^{\prime} \mathrm{C} \epsilon(2) \mathrm{G} \mathrm{C} \epsilon(8) \mathrm{BC} \epsilon(8) \mathrm{SC} \epsilon(2)$ & 20 \\
$701-703$ & $\mathrm{~S}^{\prime} \mathrm{C} \beta(5) \mathrm{B} \mathrm{C} \beta(12) \mathrm{GC} \beta(5) \mathrm{BC} \beta(9)$ & 31 \\
$718-725$ & $\mathrm{TC} \epsilon(7) \mathrm{S}^{\prime} \mathrm{C} \epsilon(2) \mathrm{GC} \epsilon(12)$ & 21 \\
$729-731$ & $\mathrm{GN} \epsilon(2) \mathrm{GN} \alpha(7) \mathrm{BN} \alpha(7)$ & 16 \\
741 & $\mathrm{D}^{\prime} \alpha(2)$ & 2 \\
$747-751$ & $\mathrm{TN} \alpha(2) \mathrm{GN} \beta(6) \mathrm{BN} \beta(2) \mathrm{SN} \beta(3)$ & 13 \\
757 & $\mathrm{TN} \epsilon(2)$ & 2 \\
777 & $\mathrm{TN} \beta(3)$ & 3 \\
783 & $\mathrm{TC} \alpha_{\mathrm{L}}(3)$ & 3 \\
\hline
\end{tabular}

\footnotetext{
${ }^{\text {a }}$ Based on selection of most frequently found conformers. See text.

b When two bands are given, italicized frequency is expected to be weaker (based on PED); otherwise, bands are expected to be of comparable intensity.

$\chi^{2} \chi^{1} \phi, \psi$ (number of examples).
}

using our ab initio disulfide force field ${ }^{3}$ combined with our empirical peptide force field. ${ }^{7}$ This approach is the most definitive way of correlating spectrum with structure of the $\mathrm{S}-\mathrm{S}$ bridge. In this paper, in order to provide some potentially useful general correlations, we have calculated the $\nu(\mathrm{SS})$ and $\nu(\mathrm{CS})$ frequencies of a range of $\mathrm{S}-\mathrm{S}$ bridge conformations. These structures all have $\chi^{3}=90^{\circ}$ (which is most commonly found) and cover the complete range of $\chi^{2}$, the rotational isomeric states of $\chi^{1}$, and the adjoining $\phi, \psi$ of the $\alpha$-helix and $\beta$ strand (on which we show a dependence for the first time). Such results (Tables I-IV) should help to predict expected $\nu(\mathrm{SS})$ and $\nu(\mathrm{CS})$ frequencies of given $\mathrm{S}-\mathrm{S}$ bridge structures.

We have also examined the potential for predicting structure from the observed spectral bands. This is more difficult, since we find that $\nu$ (SS) and $\nu$ (CS) are determined by 9 dihedral angles of the bridge whereas the above stretching modes provide at most three pieces of data. However, if we use known protein disulfide bridge structures as a representative guide, we can at least provide a statistical measure of the likely conformations to be associated with given spectral regions. In a previous paper, ${ }^{5}$ we calculated the $\nu(\mathrm{SS})$ modes of $92 \mathrm{~S}-\mathrm{S}$ bridges found in proteins and showed that they could be classified into 4 major groups of conformations according to the frequency range. The present more general calculations (Tables I-III) essentially sup- port this classification, although for some conformations (e.g., SS' and GS) there is a possibility of overlapping ranges. This further emphasizes the limitations of an earlier classification scheme based on correlations from simple model compounds. ${ }^{6}$ In this paper we have done a similar analysis of the $\nu(\mathrm{CS})$ modes (Table VI), thus providing for the possibility of a more detailed assignment of the $\chi^{2} \chi^{1}$ $\phi, \psi$ part of the bridge than was possible from model compound studies. ${ }^{6}$ The combined information provided by these more detailed $\nu$ (SS) and $\nu(\mathrm{CS})$ constraints should help identify $\mathrm{S}-\mathrm{S}$ bridge conformation more specifically than was previously possible.

In actual practice, of course, there are qualifications to the straightforward application of these relationships. Obviously, if a system has more than one $\mathrm{S}-\mathrm{S}$ bridge (as many proteins do), we have to resolve the individual contributions, which could be particularly problematic in the $\nu$ (SS) region. Another difficulty might be assigning the $\nu$ (CS) modes, since they occur in a region common to other deformation modes [in particular, $\nu(\mathrm{CS})$ of methionine ${ }^{12}$ ] and they are usually weaker than the $\nu$ (SS) modes. It might be thought that resonance Raman could be helpful in identifying these modes; it appears, however, that resonance enhancement will require excitation in the vacuum uv region. ${ }^{13}$ One possible route to identification would be through isotopic substitution. For example, we find that for 
$\mathrm{C}_{1}^{\beta}=\mathrm{C}_{2}^{\beta}={ }^{13} \mathrm{C}$ the $\nu(\mathrm{CS})$ for $\phi_{1}, \psi_{1}=\phi_{2}, \psi_{2}=\beta$ shift by 3-6 $\mathrm{cm}^{-1}$ for $\chi^{1}=\mathrm{C}$ (depending on $\chi^{2}$ ), by 8-11 $\mathrm{cm}^{-1}$ for $\chi^{1}=\mathrm{H}$, and by $11-16 \mathrm{~cm}^{-1}$ for $\chi^{1}=\mathrm{N}$. Strong inferences about structure will require such approaches, but these are made possible by the more reliable insights provided by normal mode analysis.

This research was supported by NSF grants DMB-8816756 and DMR-9110353.

\section{REFERENCES}

1. Zhao, W., Bandekar, J. \& Krimm, S. (1988) J. Am. Chem. Soc. 110, 6891-6892.

2. Zhao, W. \& Krimm, S. (1990) J. Mol. Struct. 224, $7-20$.

3. Zhao, W., Bandekar, J. \& Krimm, S. (1990) J. Mol. Struct. 238, 43-54.

4. Qian, W., Zhao, W.\& Krimm, S. (1991) J. Mol. Struct. 250, 89-102.
5. Qian, W. \& Krimm, S. (1992) Biopolymers, 32, 321326.

6. Sugeta, H., Go, A. \& Miyazawa, T. (1972) Chem. Lett. 83.

7. Krimm, S. \& Bandekar, J. (1986) Adv. Protein Chem. 38, 181-364.

8. Srinivasan, N., Sowdhamini, R., Ramakrishnan, C. \& Balaram, P. (1990) Int. J. Peptide Protein Res. 36, 147-155.

9. Tiffany, M. L. \& Krimm, S. (1968) Biopolymers 6, 1379-1382.

10. Woody, R. W. (1992) Adv. Biophys. Chem., 2, 37-79.

11. Kuptsov, A. H. \& Trofimov, V. I. (1985) J. Biomol. Struct. Dynam. 3, 185-196.

12. Nogami, N., Sugeta, H. \& Miyazawa, T. (1975) Chem. Lett., 147-150.

13. Johnson, C. R. \& Asher, S. A. (1987) J. Raman Spectroscopy 18, 345-349.

Received December 2, 1991

Accepted January 28, 1992 